\title{
Light quality on growth and phenolic compounds accumulation in Moringa oleifera L. grown in vitro
}

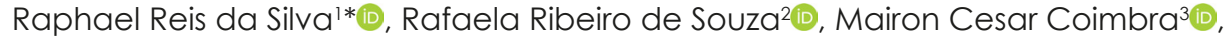 \\ Fernanda Carlota Nery ${ }^{4}$, Amauri Alves de Alvarenga ${ }^{2}{ }^{\circ}$, Renato Paiva ${ }^{2}(-)$

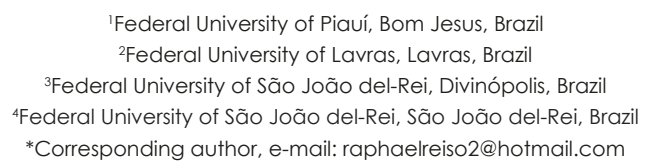

Abstract

All plant parts of the Moringa oleifera can contain relevant concentrations of phytochemicals with health benefits. Plants grown in vitro allow pathogen-free plant material production and rapid propagation, and this technique is widely used to obtain secondary metabolites. This study analyzed how light spectrum quality affects growth, chlorophyll, and total phenolic content in M. oleifera plants grown in vitro. M. oleifera seeds were inoculated in MS medium supplemented with $3 \%$ sucrose and $0.7 \%$ agar and were stored under a controlled temperature, humidity and photoperiod. The light conditions tested were white fluorescent lamps (WFL) and light-emitting diodes (LED: $70 \%$ red $+30 \%$ blue), both standardized with a photon flux density of $58 \mu \mathrm{mol} \mathrm{m} \mathrm{m}^{-2} \mathrm{~s}$ '. Radiation emitted by WFL and LED did not influence stem germination, height, or diameter. However, WFL provided higher total chlorophyll levels. All plant parts germinated in vitro were analyzed via high-performance liquid chromatography with a diode-array detector (HPLC-DAD), and preliminary analyses revealed preferential synthesis of gallic acid derivatives in the leaves under LED radiation. For the total phenolic content, leaves under the LED and WFL irradiations showed $3.524 \pm 0.054$ and $3.805 \pm 0.304$ micrograms, respectively, of gallic acid

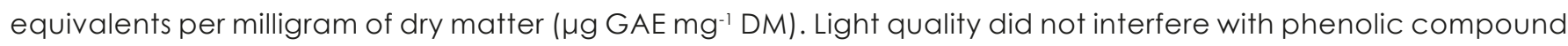
accumulation; however, it stimulated the preferential synthesis of gallic acid derivatives in leaves grown under LED radiation, and the evidence indicated that all evaluated organs synthesized nonpolar substances.

Keywords: medicinal plant, moringaceae, phytochemistry, spectral quality

\section{Introduction}

Light is one of the most important environmental factors regulating and affecting plant development and behavior because it is intrinsically related to the plant's biological processes (Zheng \& Van Labeke, 2017). Plants must be flexible in response to their light environment, and this flexibility means sensing the amount (creep rate), quality (wavelength, i.e., color), and direction and duration (photoperiod) (Topchiy et al., 2005; Bian et al., 2014; Ouzounis et al., 2015; Batista et al., 2018).

The use of light-emitting diode (LED) lamps in plant tissue culturing has been shown to be advantageous and promising, especially because it allows selecting specific regions of the light spectrum, leading to desired or hypothesized physiological responses (Topchiy et al., 2005; Mengxi et al., 2011; Bian et al., 2014; Ouzounis et al., 2015).

Moringa oleifera Lam., known as moringa, is a rustic, fast-growing plant with high levels of mineral nutrients, such as potassium and magnesium; amino acids, such as phenylalanine and L- tryptophan; organic acids, such as malic and citric acids; and phytochemicals, such as phenolic compounds (PCs), including gallic acid, rutin, and kaempferol (Manguro \& Lemmen, 2007). This species to have a range of bioactivity, including antiinflammatory, anticancer, antioxidant, and antimicrobial activity, and has been used to treat water (Barnabas et al., 2013; Vongsak et al., 2013; Kansal \& Kumari, 2014; Maldini et al., 2014; Lin et al., 2018).

In gram-to-gram comparisons, moringa can provide 10 times more vitamin A than that of carrots, 4-7 times more vitamin $C$ than that of oranges and 4-15 times more potassium than that of bananas. In addition, is rich in phytosterols such as stigmasterol and sitosterol, compounds related to estrogen production, which stimulate mammary gland growth for milk production 
(Gopalakrishnan et al., 2016). Thus, moringa has been considered the tree of life and has become an object of study in several areas, such as agriculture, livestock, animal nutrition, industry, and medicine (Kansal \& Kumari, 2014; Leone et al., 2016; Lin et al., 2018).

PCs constitute one of the main and broadest phytochemical groups with potential benefits to human health. They are characterized by a functional hydroxyl group linked to an aromatic ring. The shikimic acid pathway is the main pathway responsible for producing phenolic compounds, and phenylalanine ammonialyase is this pathway's main enzyme (Quideau et al., 2011 ; Cheynier et al., 2013).

Although studies have shown the influence of light quality on plant physiology and behavior, the light spectrum-dependent responses for each species should also be analyzed, as these responses vary greatly and may have different patterns (Batista et al., 2016; Souza et al., 2016; Li et al., 2017; Batista et al., 2018). This study analyzed how light spectrum quality affects growth, chlorophyll, and total phenolic content in M. oleifera plants grown in vitro.

\section{Material and Methods}

Plant material

The seeds used in this experiment were collected from ripe fruits of $M$. oleifera plants grown under semi-arid conditions ( $9^{\circ} 9^{\prime} \mathrm{S} 40^{\circ} 22^{\prime} \mathrm{W}, 350 \mathrm{~m}$ ) in Petrolina-PE, Brazil. The voucher specimen was deposited in the Embrapa Semiárido herbarium under accession number 7949.

\section{Asepsis and culture conditions}

First, the tegument was removed, and seeds were selected for uniformity and absence of apparent physical damage using magnifying glasses.

Selected seeds underwent asepsis in a laminar flow hood. First, the samples were immersed in $70 \%$ ethanol for 1 minute and then washed in autoclaved distilled water. Next, the seeds were immersed in commercial sodium hypochlorite solution ( $\mathrm{NaOCl} 2.5 \%$ ) for 10 minutes and then washed in autoclaved distilled water three times.

The seeds were inoculated into MS medium (Murashige \& Skoog, 1962), containing sucrose (3\%) and agar $(0.7 \%)$. The $\mathrm{pH}$ was adjusted to 5.8 before autoclaving at $120^{\circ} \mathrm{C}$ for 20 minutes.

The plant material were kept in a growth room under a controlled and monitored temperature (25 \pm 2 $\left.{ }^{\circ} \mathrm{C}\right)$, humidity $\left(70 \pm 5^{\circ} \mathrm{C}\right.$ ) and photoperiod (16/8 light/dark) and cultivated for 23 days from the date of inoculation.

The light conditions tested were white fluorescent lamps (WFL) and light-emitting diodes (LEDs, 70\% red + $30 \%$ blue), both standardized with a photon flux density of $58 \mu \mathrm{mol} \mathrm{m} \mathrm{m}^{-2} \mathrm{~s}^{-1}$ To determine the quality of light (Figure 1), a spectroradiometer (Red TIDE USB 650 UV, LICOR, USA) was used.

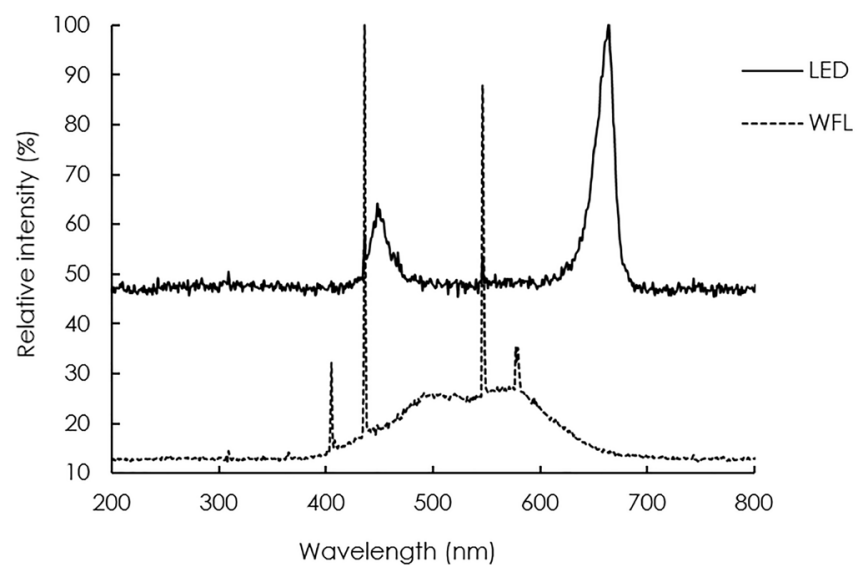

Figure 1. Spectral characteristics of lamps used as a light source for the growth of Moringa oleifera in vitro.

\section{Quantification of total chlorophyll and growth}

A nondestructive method of measuring transmittance through the leaf surface at wavelengths associated with chlorophyll was used to quantify total chlorophyll content (Zhu et al., 2012). Readings were taken in triplicate in mature leaves from 10 plants per treatment, and the value was expressed in $\mathrm{mg} \mathrm{cm}^{-2}$. In addition, growth parameters were evaluated: hypocotyl length (HL), hypocotyl diameter (DIA) and root length $(\mathrm{RL})$. This experiment was conducted in a completely randomized design, with two treatments consisting of the LED and WFL luminosity conditions.

Determination of total phenolic content

Samples of the differents plantlets organs grown in vitro (root, hypocotyl and leaf) were dried at $38 \pm 1{ }^{\circ} \mathrm{C}$ for $48 \mathrm{~h}$. Subsequently, $200 \mathrm{mg}$ of the dried samples were extracted in $70 \%$ ethanol by sonication at $35^{\circ} \mathrm{C}$ for 30 minutes, then filtered using filter paper. The volume was filled to $10 \mathrm{~mL}$ with $70 \%$ ethanol.

Aliquots of $250 \mu \mathrm{L}$ from the samples were used to determine the total phenolic compounds using the FolinCiocalteu method. Gallic acid $\left(200 \mu \mathrm{g} \mathrm{mL}^{-1}\right)$ was used as the standard solution. Determinations were performed in triplicate, and the results were expressed in micrograms of gallic acid equivalents per milligram of dry matter ( $\mu \mathrm{g}$ GAE mg $\left.{ }^{-1} \mathrm{DM}\right)$. The experiment was performed in a completely randomized factorial design of 2 (ligth quality) $\times 3$ (organs). Fifteen biological replicates were evaluated per treatment, and each replicate consisted of five plants. 
High-performance liquid chromatography with diode array detection (HPLC-DAD)

Samples were analyzed using the Shimadzu Prominence UFLC modular liquid chromatography system (Shimadzu Corp., Kyoto, Japan) and a computerized system operated by LC WorkStation software (LC Solution, version 1.25). The dried extract was then dissolved in methanol (1 $\mathrm{mg} \mathrm{mL}^{-1}$ ) and filtered through a $0.45 \mu \mathrm{m}$ polytetrafluoroethylene (PTFE) syringe filter (MachereyNagel, Bethlehem, PA, USA). Compounds were separated in a C18 Gemini reverse-phase column $(4.6 \times 250 \mathrm{~mm}, 5$ $\mu \mathrm{m}$ particle size) with a $4.0 \times 2.0 \mathrm{~mm}$ i.d. guard column of the same material (both from Phenomenex®, Torrance, CA, USA) at $35{ }^{\circ} \mathrm{C}$. The mobile phases used were $\mathrm{A}$ : water:formic acid (99.9:0.1) and B: methanol:formic acid (99.9:0.1), in proportions of 0\% B (0-5 minutes), 0-100\% B (5-30 minutes), and 100\% B (30-35 minutes). A $20-\mu \mathrm{L}$ injection volume and a $1.0-\mathrm{mL} \mathrm{min}^{-1}$ flow were used. Separations were monitored at three wavelengths (254 nm, $330 \mathrm{~nm}$, and $350 \mathrm{~nm}$ ) to detect more phenolic substances. Phenolic compounds present in the samples were determined by comparing the retention times and spectra obtained via the previously injected patterns with data reported in the literature.

\section{Statistical analyses}

The data were subjected to analysis of variance using the statistical software ASSISTAT 7.7 (Silva \& Azevedo, 2016), to diagnose significant effects by the $F$ test, and the averages were compared by the Tukey's test $(\mathrm{P}<$ 0.05 and 0.011 .

\section{Results}

Growth

During the experiment, the seeds were evaluated daily for root protrusion. Light quality not interfered significantly in this evaluation, and an average of five days was needed for radicle protrusion in both treatments.

Light quality did not significantly affect $\mathrm{HL}$ or DIA; however, the radiation provided by the LED lamps yielded an increase RL of $1.29 \mathrm{~cm}$ (Table 1). Chlorophyll content

Table 1. Means of hypocotyl length (HL), root length (RL), and hypocotyl diameter (DIA) of Moringa oleifera plantlets grown in vitro in different light environments.

\begin{tabular}{|c|c|c|c|}
\hline Treatments & $\mathrm{HL}(\mathrm{cm}) \pm \mathrm{SE}$ & $\mathrm{RL}(\mathrm{cm}) \pm \mathrm{SE}$ & $\mathrm{DIA}(\mathrm{mm}) \pm \mathrm{SE}$ \\
\hline LED & $10.28 \pm 0.18 \mathrm{~ns}$ & $7.45 \pm 0.19 a$ & $2.87 \pm 0.05 \mathrm{~ns}$ \\
\hline WFL & $10.67 \pm 0.24 \mathrm{~ns}$ & $6.16 \pm 0.16 b$ & $3.02 \pm 0.05 \mathrm{~ns}$ \\
\hline $\mathrm{CV}(\%)$ & 12 & 15 & 17 \\
\hline
\end{tabular}

Light quality is an important factor for photosynthetic pigment biosynthesis. Irradiance from WFL provided a higher total chlorophyll content, with an approximately $14 \%$ increase compared with LED (Figure 2).

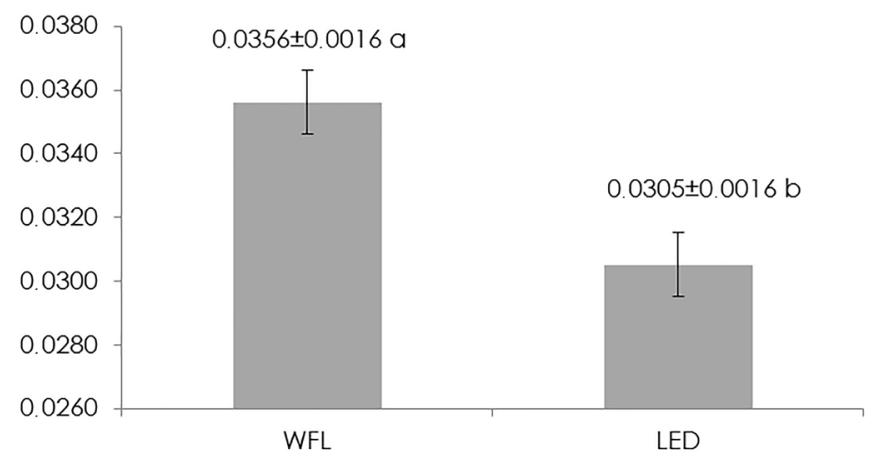

Figure 2. Total chlorophyll ( $\mathrm{mg} \mathrm{cm}^{-2}$ ) in Moringa oleifera leaves grown in vitro under different light conditions. Data are represented as the mean \pm standard error. Means followed by the same letter do not differ by the Tukey's test $(P<0.05)$.

Total phenolic content

There was a significant interaction in the factors organs and light quality for the content of total phenolics. Where, it is observed that the leaves, regardless of the light quality treatment, presented higher concentrations of total phenolics compared to the hypocotyl and root organ (Table 2). Regarding the effect of light quality, it is observed that the hypocotyl and root showed the same pattern of accumulation that resulted in higher concentrations over white light (Table 2).

Table 2. Mean values \pm standard deviation for total phenolic content in micrograms of gallic acid equivalents per milligram of dry matter ( $\mu \mathrm{g} \mathrm{GAE} \mathrm{mg}{ }^{-1} \mathrm{DM}$ ) in the leaves, hypocotyls, and roots of Moringa oleifera plantlets grown in vitro under different light conditions.

\begin{tabular}{cccc}
\hline \multicolumn{4}{c}{ Organ } \\
\hline Light quality & Leaf & Hypocotyl & Root \\
LED & $3.524 \pm 0.054 \mathrm{aA}$ & $2.565 \pm 0.187 \mathrm{bB}$ & $2.185 \pm 0.089 \mathrm{bB}$ \\
WFL & $3.805 \pm 0.304 \mathrm{aA}$ & $3.303 \pm 0.246 \mathrm{aB}$ & $3.180 \pm 0.063 \mathrm{aB}$ \\
\hline CV (\%) & 6.02 & 6.02 & 6.02 \\
\hline Means followed by the same letter do not differ among themselves by the Tukey's test $(P<0.05)$.
\end{tabular}

The capital letters compare the different light quality, and the lowercase letters compare the organs.

\section{HPLC-DAD}

The chromatograms presented refer to tests performed on the leaves. This procedure was performed on all samples; however, the separation method required adjustments, which are being provided. Furthermore, the chromatograms from all samples suggested that M. oleifera plants produced more nonpolar than polar substances.

Based on retention time and wavelength, leaves grown under LED radiation yielded a preferential synthesis of gallic acid derivatives (Figures 3 and 4); however, this pattern was not observed in leaves grown under WFL at a wavelength of $205 \mathrm{~nm}$. 
A)

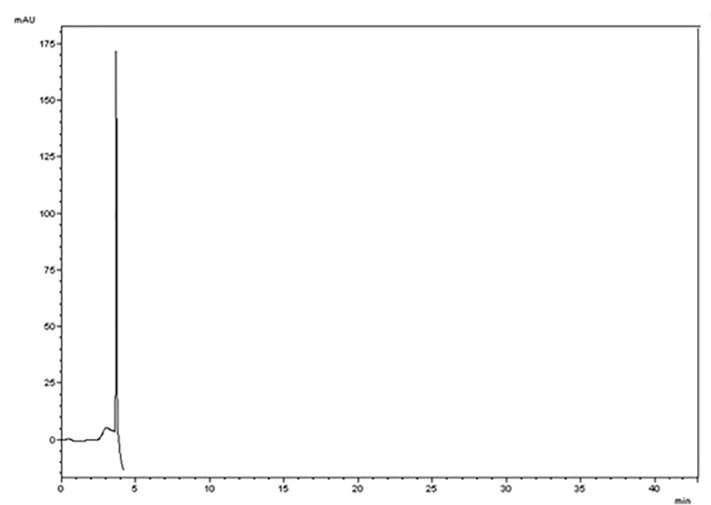

B)

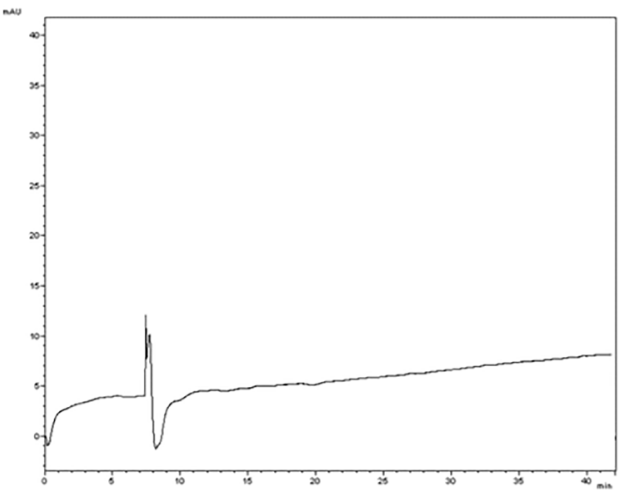

Figure 3. Chromatograms obtained by HPLC-DAD analysis of Moringa oleifera leaves grown in vitro under different light conditions. LED (a) and WFL (b). Detection at $254 \mathrm{~nm}$.

A)

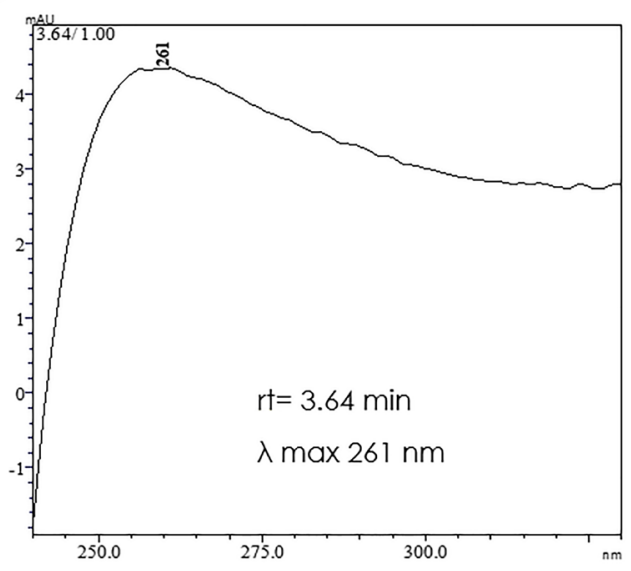

B)

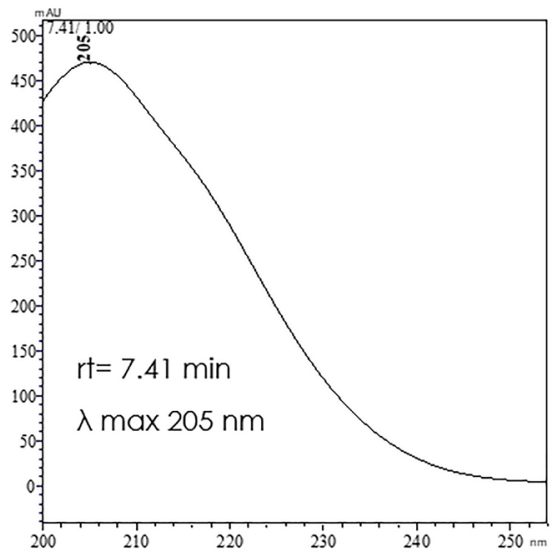

Figure 4. UV-visible spectra for peaks identified in the chromatogram of Moringa oleifera leaves grown in vitro under different light conditions. LED (a) and WFL (b). Detection at $254 \mathrm{~nm}$. Legend: r† $=$ retention time in minutes; $\lambda$ max $=$ wavelength with the highest absorbance.

\section{Discussion}

Chlorophyll content and growth

The data showed that differences in light quality yielded different responses in total chlorophyll, root length (Table 1 and Figure 2).

Regarding the total chlorophyll content, we observed that the leaves grown under WFL showed higher total chlorophyll content than did leaves grown under LED. Light quality plays an important role in photosynthesis, as it influences the way in which light is absorbed by photosynthetic pigments (Topchiy et al., 2005). There are reports that demonstrate that blue light is important in chlorophyll biosynthesis and maturation of chloroplasts. Which may indicate that the higher relative intensity of blue light present in the WFL influenced the biosynthesis of this pigment (Figure 1). In contrast, Batista et al. (2016) observed a different response regarding total chlorophyll content in Lippia alba chemotypes, where higher photosynthetic pigment levels were observed in leaves grown under LED (blue/red).

As for the root length, we observed that the RL of plantlets grown in LED was significantly higher than that of plantlets grown in WFL. This difference can be attributed to a photomorgenic and gene expression response triggered by the red light provided by the LED, since it plays an important role in the regulation of root growth (Li et al., 2017; Zheng \& Van Labeke, 2017; Batista et al., 2018). In addition, the combination of red and blue light (70\% red and $30 \%$ blue) provided by LEDs has also shown to have an effect on promoting the root lenght of Oncidium plantlets produced in vitro (Mengxi et al., 2011).

\section{Total phenolic content and HPLC-DAD}

The content of total phenolic compounds showed a pattern of differential accumulation in the different organs studied, with a higher concentration in leaves (Table 2). Several studies have also shown that the synthesis and accumulation of secondary metabolites varies between species, organs, tissues and cells (Kim et al., 2015; Batista et al., 2018). The regulatory pathways of synthesis, transport and accumulation of secondary metabolites are complex and diverse in the different 
organs. The leaves, in addition to playing an important role in the life cycle of vegetables as they are the main organs of the plant responsible for photosynthesis, can also be used as an MS storage organ (Li et al., 2020). Which indicates that in $M$. oleifera in the initial development stages, the leaves are prioritized as the main storage organ for total phenolics.

Additionally, it was verified through the HPLC analysis that leaves under LED light showed preferential synthesis of gallic acid derivatives (Figures 3 and 4). Gallic acid is a secondary metabolite found in plants, which exhibits a range of bioactivities such as antioxidant, antimicrobial, anti-inflammatory, antitumor, antibacterial and anticarcinogenic activities (Sakakibara et al., 2003; Manguro \& Lemmen, 2007; Coimbra et al., 2017; Fernandes \& Salgado, 2016). Secondary metabolites, especially PCs, are involved in plant responses to biotic and abiotic stresses and can contribute significantly to the plant's antioxidant activity. In addition, these metabolites have been identified as important allies to human health because they can aid in preventing and treating diseases (Manguro \& Lemmen, 2007; Coimbra et al., 2017).

Metabolite biosynthesis, in addition to presenting specificity in relation to tissue and organ, is also regulated by environmental conditions, and its levels may be increased, thus increasing the yields of various compounds or classes of metabolites of medicinal interest. Thus, in vitro cultivation is a very useful and successfully exploited tool, either to provide a better understanding of the biological mechanisms involved between the physical environment and plants or to increase the metabolic levels or specificity (Ouzounis et al., 2015; Batista et al., 2016; Souza et al., 2016).

The quality of light is one of the environmental factors that most influenced the balance of primary and secondary metabolites in plants, and in our results, we observed that the quality of the light provided by the WFL played an important role in obtaining a higher content of total phenolics in hypocotyl and roots of $M$. oleifera plantlets (Table 2). Root and hypocotyl are also organs of accumulation of active compounds and this accumulation was differential due to the light quality provided by LEDs and WFL. This differential accumulation probably occurred due to an induction of the phenylpropanoid biosynthetic pathway caused by blue light (Kim et al., 2015). Since, the intensity of blue light contained in white light is higher (Figure 1).

The information obtained in this research is extremely relevant and may provide support for future research aimed at obtaining greater content of bioactive phenolic compounds in plants of M. oleifera. However, understanding the regulatory biochemical mechanisms that control the types and amounts of phenolic compounds produced under different experimental conditions in the most diverse tissues or organs of plants is still a challenge.

\section{Conclusions}

Light quality did not affect phenolic compound accumulation in $M$. oleifera leaves; however, it stimulated preferential synthesis of gallic acid derivatives in leaves grown under LED radiation in addition to providing greater root growth as observed in plants grown under LED radiation. These results may open new possibilities for exploring large-scale production of gallic acid derivatives.

\section{Acknowledgment}

The authors would like thanks to their collaborators and CNPq, CAPES and FAPEMIG for the financial support.

\section{References}

Barnabas, J., Murthy, S.S., Jagdeesh. 2013. Antimicrobial properties of endophytic fungi isolated from Cynodon dactylon and Moringa oleifera. International Journal of Biological \& Pharmaceutical Research 4: 98-104.

Batista, D.S., Castro, K.M., Silva, A.R., Teixeira, M.L., Sales, T.A., Soares, L.I., Cardoso, M.G., Santos, M.O., Viccini, L.V., Otoni, W.C. 2016. Light quality affects in vitro growth and essential oil profile in Lippia alba (Verbanaceae). In Vitro Cellular \& Developmental Biology - Plant 52: 276-282.

Batista, D.S., Felipe, S.H.S., Silva, T.D., Castro, K.M., Mamedes-Rodrigues, T.C., Miranda, N.A., Ríos-Ríos, A.M., Faria, D.V., Fortini, E.A., Chagas, K., Torres-Silva, G., Xavier, A., Arencibia, A.D., Otoni, W.C. 2018. Light quality in plant tissue culture: does it matter? In Vitro Cellular \& Developmental Biology - Plant 54: 195-215.

Bian, Z.H., Yang, Q.C., Liu, W.K. 2014. Effects of light on the accumulation of phytochemicals in vegetables produced in controlled environments: a review. Journal of the Science of Food and Agriculture 95: 869-877.

Cheynier, V., Comte, G., Davies, K.M., Lattanzio, V., Martens, S. 2013. Plant phenolics: Recent advances on their biosynthesis, genetics, and ecophysiology. Plant Physiology and Biochemistry 72: 1-20.

Coimbra, M.C., Chagas, R.C.R., Duarte-Almeida, J.M., Castro, A.H.F. 2017. Influence of plant growth regulators and light on callus induction and bioactive phenolic compounds production in Pyrostegia venusta (Bignoniaceae). Indian Journal of Experimental Biology 55: $584-590$.

Fernandes, S.H.A., Salgado, H.R.N. 2015. Gallic acid: Review of the methods of determination and quantification. Critical Reviews in Analytical Chemistry 46: 257-265. 
Gopalakrishnan, L., Doriya, K., Kumar, D.S. 2016. Moringa oleifera: A review on nutritive importance and its medicinal application. Food Science and Human Wellness 5: 49-56.

Kansal, K.S., Kumari, A. 2014. Potential of M. oleifera for the treatment of water and wastewater. Chemical Reviews 114: 4993-5010.

Kim, Y.J., Kim, Y.B., Li, X., Choi, S.R., Park, S., Park, J.S., Lim, Y.P., Park, S.U. 2015. Accumulation of phenylpropanoids by white, blue, and red light irradiation and their organspecific distribution in chinese cabbage (Brassica rapa ssp. Pekinensis). Journal of Agricultural and Food Chemistry 63: 6772-6778.

Leone, A., Spada, A., Battezzati, A., Schiraldi, A., Aristil, J., Bertoli, S. 2016. Moringa oleifera seeds and oil: characteristics and uses for human health. International Journal of Molecular Sciences 17: 1-14.

Li, C.X., Xu, Z.H., Dong, R.Q., Chang, S.X., Wang, L.Z., KhalilUr-Rehman, M., Tao, J.M. 2017. An RNA-seq analysis of grape plantlets grown in vitro reveals different responses to blue, green, red LED light, and white fluorescent light. Frontiers in Plant Science 8: 78.

Li, Y., Kong, D., Fu, Y., Sussman, M.R., Wu, H. 2020. The effect of developmental and environmental factors on secondary metabolites in medicinal plants. Plant Physiology and Biochemistry 148: 80-89.

Lin, M., Zhang, J., Chen, C. 2018. Bioactive flavonoids in Moringa oleifera and their health-promoting properties. Journal of Functional Foods 47: 469-479.

Maldini, M., Maksoud, S.A., Natella, F., Montoro, P., Petretto, G.L., Foddai, M., Nicola, G.R., Chessa, M. Pintore, G. 2014. 'Moringa oleifera: study of phenolics and glucosinolates by mass spectrometry'. Journal Mass Spectrometry 49: 900-910.

Manguro, L.O.A., Lemmen, P. 2007. Phenolics of Moringa oleifera leaves. Natural Product Research 21: 56-68.

Mengxi, L., Zhigang, X., Yang, Y., Yijie, F. 2011. Effects of different spectral lights on Oncidium PLBs induction, proliferation, and plant regeneration. Plant Cell, Tissue and Organ Culture 106: 1-10.

Murashige, T., Skoog, F. 1962. A revised medium for rapid growth and bio assays with tobacco tissue plants. Physiologia Plantarum 15: 473-497.

Ouzounis, T., Rosenqvist, E., Ottosen, C.-L. 2015. Spectral effects of artificial light on plant physiology and secondary metabolism: a review. HortScience 50: 1128-1135.

Quideau, S., Deffieux, D., Douat-Casassus, C., Pouységu, L. 2011. Plant polyphenols: chemical properties, biological activities, and synthesis. Angewandte Chemie International Edition 50: 586-621.

Sakakibara, H., Honda, Y., Nakagawa, S., Ashida, H., Kanazawa, K. 2003. Simultaneousdetermination of all polyphenols in vegetables, fruits, and teas. Journal of Agricultural and Food Chemistry 51: 571-581.
Silva, F.A.S., Azevedo, C.A.V. 2016. The Assistat software version 7.7 and its use in the analysis of experimental data. African Journal of Agricultural Research 11:3733-3740.

Souza, R.R., Paiva, P.D.O., Silva, R.R., Reis, M.V., Nery, F.C., Paiva, R. 2016. Optimization of jenipapo in vitro seed germination process. Ciência e Agrotecnologia 40: 658664.

Topchiy, N.M., Sytnik, S.K., Syvash, O.O., Zolotareva, O.K. 2005. The effect of additional red irradiation on the photosynthetic apparatus of Pisum sativum. Photosynthetica 43: 451-456.

Vongsak, B., Sithisarna, P., Mangmool, S., Thongpraditchotec, S., Wongkrajangc, Y., Gritsanapana, W. 2013. Maximizing total phenolics, total flavonoids contents and antioxidant activity of Moringa oleifera leaf extract by the appropriate extraction method. Industrial Crops and Products 44: 566-571.

Zheng, L., Van Labeke, M.-C. 2017. Long-term effects of red- and blue-light emitting diodes on leaf anatomy and photosynthetic efficiency on three ornamental plants. Frontiers in Plant Science 8: 917.

Zhu, J., Tremblay, N., Liang, Y. 2012. Comparing SPAD and atLEAF values for chlorophyll assessment in crop species. Canadian Journal of Soil Science 92: 645-648.

Conflict of Interest Statement: The authors declare that the research was conducted in the absence of any commercial or financial relationships that could be construed as a potential conflict of interest.

All the contents of this journal, except where otherwise noted, is licensed under a Creative Commons Attribution License attribuition-type BY. 